\title{
Photoluminescence On/Off Switching of a Single Colloidal Quantum Dot Using Photochromic Diarylethene
}

Nanoka Yano ${ }^{1}$, Mitsuaki Yamauchi ${ }^{1}$, Daichi Kitagawa ${ }^{2}$, Seiya Kobatake ${ }^{2}$, and Sadahiro $\operatorname{Masuo}^{1 *}$

${ }^{1}$ Department of Applied Chemistry for Environment, Kwansei Gakuin University, 2-1

Gakuen, Sanda, Hyogo 669-1337, Japan.

${ }^{2}$ Department of Applied Chemistry, Graduate School of Engineering, Osaka City

University, 3-3-138 Sugimoto, Sumiyoshi-ku, Osaka 558-8585, Japan. 


\section{Synthesis of CdSe/ZnS QDs}

Chemicals: Cadmium oxide (CdO, 99.99\%), trioctylphosphine oxide (TOPO), hexadecylamine (HDA, 95\%), tetradecylphosphonic acid (TDPA, 97\%), selenium (Se), 1-octadecene (90\%), hexane, EtOH, acetone, zinc oxide ( $\mathrm{ZnO}, 99 \%)$, sulfur ( $\mathrm{S}, 98 \%)$, oleic acid (90\%), and oleylamine (70\%).

Synthesis of CdSe core QD ${ }^{1-2}$ : TOPO (3.0 g, $\left.8.0 \mathrm{mmol}\right)$, HDA (2.0 g, $\left.8.0 \mathrm{mmol}\right)$, TDPA (0.28 g, $1 \mathrm{mmol})$, and $\mathrm{CdO}(64 \mathrm{mg}, 0.5 \mathrm{mmol})$ were loaded into a $100 \mathrm{~mL}$ three-necked flask. The mixture was heated to $100{ }^{\circ} \mathrm{C}$, degassed at temperature under vacuum for 20 min, heated to $300{ }^{\circ} \mathrm{C}$ under an argon atmosphere and stirred for $60 \mathrm{~min}$. When the $\mathrm{CdO}$ dissolved to create an optically clear solution, the temperature was adjusted to $270{ }^{\circ} \mathrm{C}$. In another $50 \mathrm{~mL}$ three-necked flask, Se $(32 \mathrm{mg}, 0.4 \mathrm{mmol})$ and $2 \mathrm{~mL}$ of 1-octadecene were mixed and degassed at $20^{\circ} \mathrm{C}$ under vacuum for $30 \mathrm{~min}$ and then placed under Ar. $2 \mathrm{~mL}$ of the Se suspension was swiftly injected into the prepared $100 \mathrm{~mL}$ three-necked flask loaded with the clear $\mathrm{Cd}$ solution at $270{ }^{\circ} \mathrm{C}$. After reacting for $1 \mathrm{~min}$ at $250{ }^{\circ} \mathrm{C}$ under an Ar atmosphere, $5 \mathrm{~mL}$ of hexane was added to the mixture to quench the reaction. The resulting suspension was separated by centrifugation $\left(5000 \mathrm{rpm}, 5 \mathrm{~min}, 20{ }^{\circ} \mathrm{C}\right)$ to remove aggregated QDs and insoluble impurities. EtOH was added to the supernatant until the 
homogeneous solution became a suspension, and the suspension was separated by centrifugation $\left(5000 \mathrm{rpm}, 5 \mathrm{~min}, 20^{\circ} \mathrm{C}\right)$ to obtain size-separated QDs as precipitates. The QDs were redispersed in $\mathrm{CHCl}_{3}$ (for spectrochemical analysis) and stored at 5-10 ${ }^{\circ} \mathrm{C}$. The diameter of the synthesized CdSe QD was estimated to be $3.2 \pm 0.2 \mathrm{~nm}$ from transmission electron microscope (TEM) image shown in Figure S1(b).

Synthesis of CdSe/ZnS core/shell QD $^{3}$ : Precursor solutions of $\mathrm{Zn}$ and $\mathrm{S}$ were prepared as follows. The precursor solution od $\mathrm{Zn}$ was prepared by mixing $\mathrm{ZnO}$ (16 $\mathrm{mg}, 0.20$ mmol) in oleic acid $(2 \mathrm{~mL})$ and 1-octadecene $(4 \mathrm{~mL})$ at $190{ }^{\circ} \mathrm{C}$ under an $\mathrm{Ar}$ atmosphere for 60 min to create an optically clear Zn-oleate solution. The precursor solution of S was prepared by mixing $\mathrm{S}(6.4 \mathrm{mg}, 0.20 \mathrm{mmol})$ in 1 -octadecene $(3 \mathrm{~mL})$ at $100{ }^{\circ} \mathrm{C}$ under an $\mathrm{Ar}$ atmosphere for $10 \mathrm{~min}$ to create an optically clear $\mathrm{S}$ solution. These mixing processes were carried out after degassing the solutions at $100{ }^{\circ} \mathrm{C}$ under reduced pressure for 10 min. To the $100 \mathrm{~mL}$ three-necked flask, $\mathrm{CHCl}_{3}$ solution of $\mathrm{CdSe}$ core QDs with $3.2 \mathrm{~nm}$ in diameter $(1 \mathrm{~mL}, 0.30 \mu \mathrm{mol})$, oleylamine $(1 \mathrm{~mL})$ and 1 -octadecene $(4 \mathrm{~mL})$ were loaded and degassed under reduced pressure at $100{ }^{\circ} \mathrm{C}$ for $30 \mathrm{~min}$, and then, the mixture was blanketed with Ar. After the temperature was increased to $245{ }^{\circ} \mathrm{C}$, the first injection of the $\mathrm{Zn}$ precursor solution $(3 \mathrm{~mL})$ was carried out. After $5 \mathrm{~min}$, the $\mathrm{S}$ precursor solution $(1.5 \mathrm{~mL})$ was injected. The $\mathrm{Zn}$ and $\mathrm{S}$ precursor solutions were then successively injected 
at 5 min intervals. After the reaction, the mixture was cooled to room temperature, and EtOH was added to the suspension, which was separated by centrifugation (5000 rpm, 5 $\min , 20{ }^{\circ} \mathrm{C}$ ). The resulting precipitate was dissolved in $\mathrm{CHCl}_{3}$ and reprecipitated with acetone, followed by separation by centrifugation $\left(5000 \mathrm{rpm}, 5 \mathrm{~min}, 20^{\circ} \mathrm{C}\right)$ to obtain sizeseparated QDs as precipitates. The QDs were redispersed in $\mathrm{CHCl}_{3}$ (for spectrochemical analysis) and stored at 5-10 ${ }^{\circ} \mathrm{C}$. The diameter of the synthesized CdSe QD was estimated to be $4.7 \pm 0.2 \mathrm{~nm}$ from TEM image shown in Figure S1(c). As the thickness of $\mathrm{ZnS}$ monolayer is $0.31 \mathrm{~nm}$, the CdSe core was covered by two monolayers of $\mathrm{ZnS}$ shells.
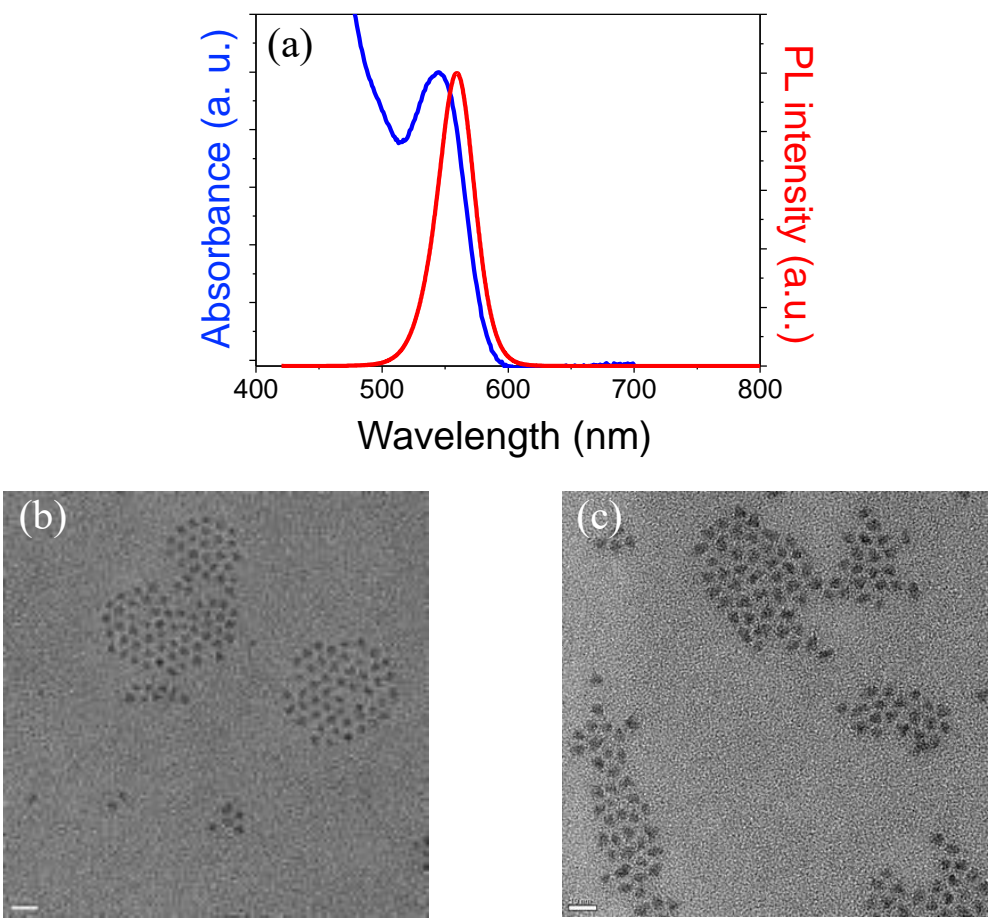

Figure S1. (a) Absorption and PL spectra of the synthesized CdSe/ZnS QD in $\mathrm{CHCl}_{3}$. (b)(c) TEM images of CdSe QD (b) and CdSe/ZnS core/shell QD (c). The scale bars in the images represent $10 \mathrm{~nm}$. 


\section{Instrument setup for the PL ON/OFF switching of single QDs}

The PL behaviors, i.e., the time trace of PL intensity, PL decay curve, and photon correlation of isolated QDs with the photoisomerization of DAE were measured using a sample-scanning inverted confocal microscope (Olympus, IX-71) in combination with a ps-pulsed laser excitation at $405 \mathrm{~nm}(10.0 \mathrm{MHz}, 90 \mathrm{ps}$ full width at half maximum; PicoQuant). The laser beam was converted to circularly polarized beam by passing a Gran-Thomson polarizer and a $\lambda / 4$ wave plate. The laser beam was introduced to the inverted microscope and reflected by a dichroic mirror (Semrock, Di01-R405) in the microscope. Then, the laser beam was focused to a diffraction-limited spot on the sample by an objective lens (Olympus, $100 \times$, NA 1.4) and irradiated to the isolated single NC. The PL emission photons from the single NC were collected by the same objective lens and passed through a confocal pinhole $(100 \mu \mathrm{m})$ with a long-pass (Semrock, LP02442RU) to remove the excitation laser. Subsequently, half of the photons were detected by a spectrometer (Acton Research Corporation, SpectraPro2358) equipped with a cooled CCD camera (Princeton Instruments, PIXIS400B). The remaining half of the photons were divided equally by a 50/50 nonpolarizing beam splitter cube into two beam paths and then detected by two avalanche single-photon counting modules (APD: PerkinElmer, SPCM-AQR-14). The signals from both APDs were connected to the router of a time- 
correlated single-photon counting (TCSPC) PC board (Becker \& Hickl, SPC630) to measure the PL lifetime and to collect data for the photon correlation histogram. The signal from one of the two APDs was delayed using a delay generator (Stanford Research, DG535) to compensate for the dead time of the TCSPC board. Time-resolved data were acquired using a first-in-first-out mode, in which the arrival time after the beginning of the acquisition, the time delay between the start and stop pulses, and the detection channel were registered for each detected PL photon. The data were analyzed using a homemade LabVIEW routine that allowed for the simultaneous measurement of PL intensity trajectory, PL lifetime, and the photon correlation histogram of single NCs. The timeresolution of the lifetime measurement, i.e., the instrumental response function (IRF) of the system, was estimated by the deconvolution of the fluorescence decay curve of erythrosine in water, which has a reported fluorescence lifetime of $87 \mathrm{ps.}{ }^{4}$ The estimated IRF was approximately $0.3 \mathrm{~ns}$.

For the wide-filed imaging of isolated QDs with the photoisomerization of DAE, continuous wave laser (405 nm, CrystaLaser) as an excitation light was introduced to the inverted microscope (Olympus, IX-71) and focused onto the sample with $30 \mu \mathrm{m}$ in diameter through the objective lens (Olympus, $100 \times$, NA 1.4). PL image of isolated QDs 
was recorded using EM-CCD camera (Hamamatsu photonics, ImagEM X2) by passing through the long-pass (Semrock, LP02-442RU) to remove the excitation laser.

\section{Determination of the UV and Vis irradiation times to observe the photoswitching behavior of single QDs}

To induce the photoisomerization of DAE in a PMMA film using LED lamps (UV; 300 $\mathrm{nm}$ with $100 \mu \mathrm{W} / \mathrm{cm}^{2}$ at sample surface, Vis; $630 \mathrm{~nm}$ with $150 \mathrm{~mW} / \mathrm{cm}^{2}$ at sample surface), the suitable irradiation times were determined by observing the absorption spectra of the DAE upon UV and Vis irradiation. The sample was prepared by drop-casting the toluene solution containing DAEs and PMMA (concentration of DAE was corresponding to Conc. 3 in main text) onto a clean coverslip and dried. Figure S2(a) shows the absorption spectra upon UV irradiation. Before UV irradiation, the open-ring isomer, DAE(o), showed no absorption at the visible range. Upon UV irradiation, the absorption bands at $380 \mathrm{~nm}$ and $585 \mathrm{~nm}$ appeared by the formation of closed-ring isomer, DAE(c). The change of the absorbance at $585 \mathrm{~nm}$ against the UV irradiation time is plotted in Figure S2(b). The absorbance reached the maximum value, i.e., photostationary state (PSS), upon UV irradiation for approximately $20 \mathrm{~s}$. Upon UV irradiation for $10 \mathrm{~s}$, the absorbance reached $88 \%$ of that at PSS. 
Figure S2(c) shows the absorption spectral change upon Vis irradiation onto the sample after UV irradiation for $60 \mathrm{~s}$. Upon Vis irradiation, the absorption of DAE(c) decreased, which indicated the photoisomerization from $\mathrm{DAE}(\mathrm{c})$ to $\mathrm{DAE}(\mathrm{o})$. The change of the absorbance at $585 \mathrm{~nm}$ against the Vis irradiation time is plotted in Figure S2(d). Although the absorption was decreased to 0.033 upon Vis irradiation for $30 \mathrm{~s}$, the absorbance did not return to 0 even by the longer irradiation. In the absorption spectra shown in Figure $\mathrm{S} 2$ (c)(inset), the broad spectra with a peak at $550 \mathrm{~nm}$ were observed even upon the longtime irradiation, for examples, $50 \mathrm{~s}, 60 \mathrm{~s}$. The origin of the absorption spectrum is attributed to the byproduct during the photoisomerization from DAE(o) upon UV irradiation. ${ }^{5}$ We observed the decrease of the formation of the by-product by decreasing the UV irradiation time. Since the by-product could be an energy acceptor of QD, short UV irradiation time was better to observe the PL photoswitching behavior of single QDs. Therefore, we selected the UV irradiation time below $10 \mathrm{~s}$. On the other hand, Vis irradiation does not damage to the DAE(c). Thus, we selected the Vis irradiation time for $60 \mathrm{~s}$ due to the efficient photoisomerization of $\mathrm{DAE}(\mathrm{c})$ to $\operatorname{DAE}(\mathrm{o})$. 

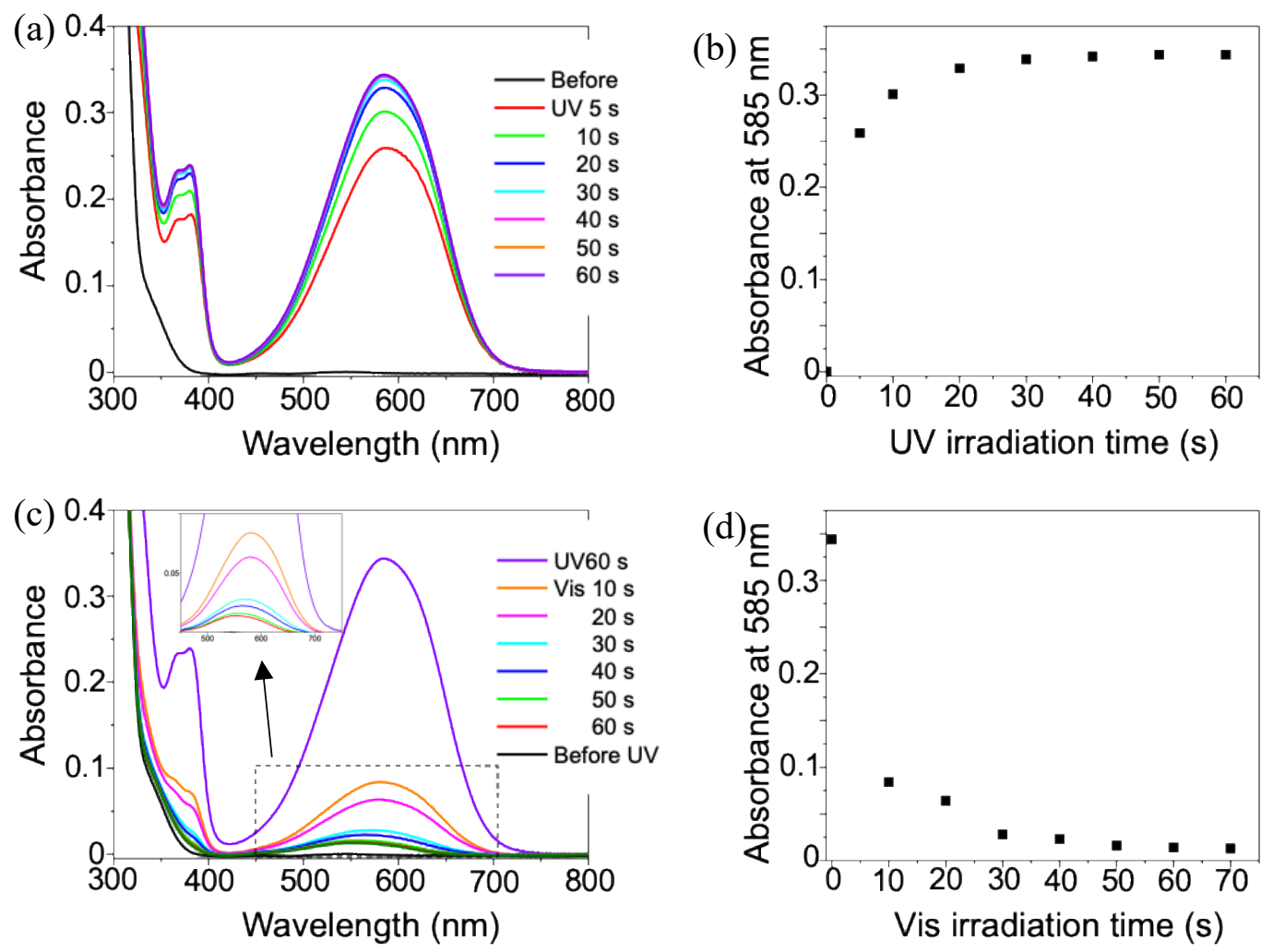

Figure S2. (a) Absorption spectra of DAE in a PMMA film upon UV irradiation. (b) Absorbance at $585 \mathrm{~nm}$ vs. UV irradiation time. (c) Absorption spectra of DAE in a PMMA film upon Vis irradiation after UV irradiation for $60 \mathrm{~s}$. A inset is expanded view of the surrounded area. (d) Absorbance at $585 \mathrm{~nm}$ vs. UV irradiation time.

\section{PL ON/OFF switching behavior of single QDs upon UV and Vis irradiation}

The PL switching behavior strongly depended on the QDs. Here, representative switching behaviors, i.e., time traces and decay curves, obtained from three single QDs using Conc. 
$\mathbf{2}$ and Conc. 3 are shown in Figure S3. The PL lifetimes before UV irradiation $\left(\tau_{\mathrm{ini}}\right)$, after UV irradiation ( $\left.\tau_{\mathrm{UV}}\right)$, and after Vis irradiation $\left(\tau_{\mathrm{Vis}}\right)$ are shown in the Figures. The FRET efficiency, i.e., shortening of the lifetime upon UV irradiation, and recovery upon Vis irradiation were strongly dependent on the QDs. The obtained PL lifetime from these single QD measurements are summarized in Figure 4 and Table 1 in main text.
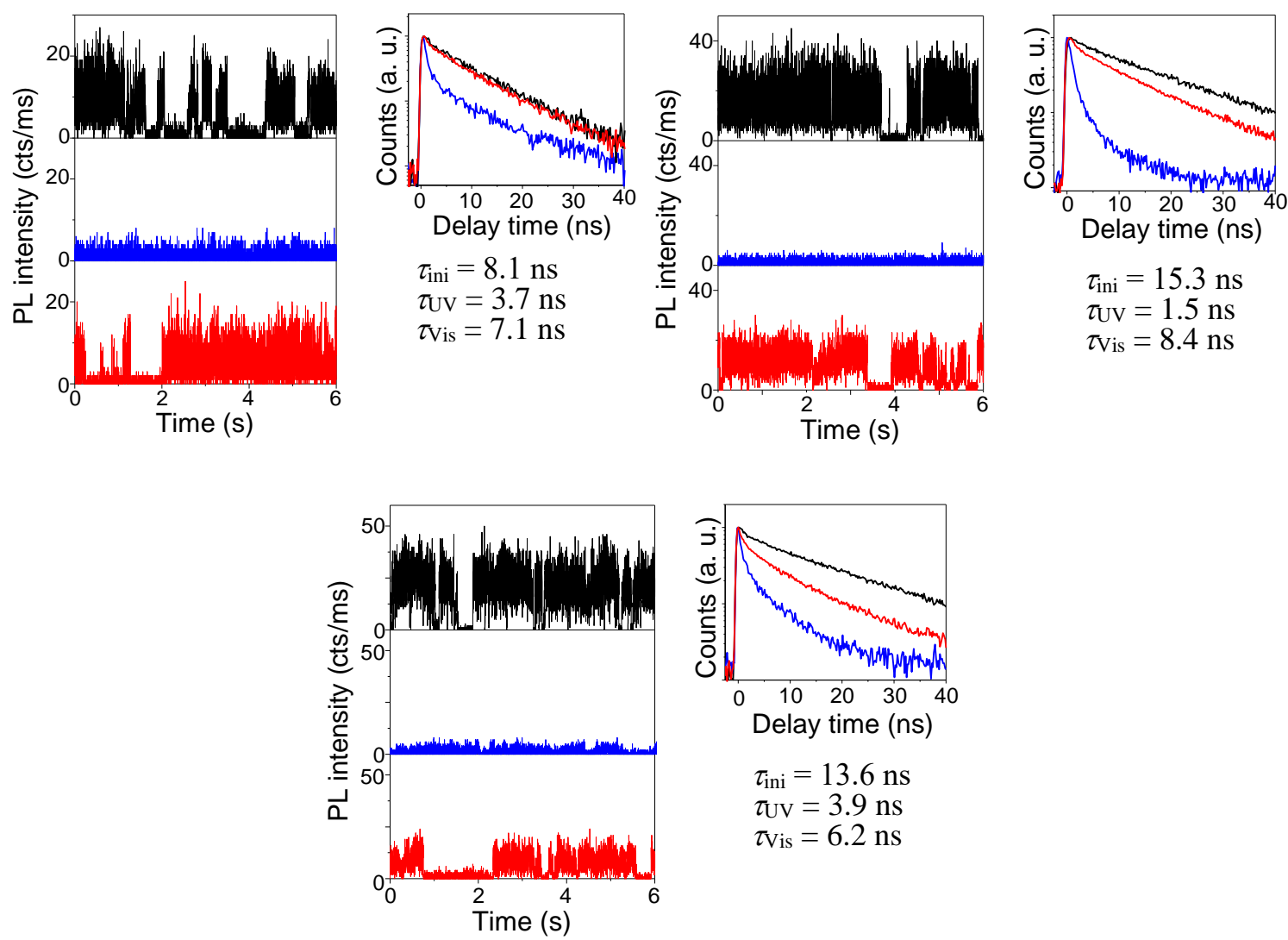

Figure S3. Time traces of the PL intensity and PL decay curves obtained from three single QDs upon UV and Vis irradiation. Black line: before UV irradiation, blue line: after UV irradiation, red line: after Vis irradiation. 
Table S1. Average PL lifetimes of single QDs upon different UV irradiation time.

\begin{tabular}{cccc}
\hline & \multicolumn{3}{c}{ Average lifetime (ns) } \\
& Conc.1 & Conc. 2 & Conc.3 \\
\hline Before & - & $13.4 \pm 2.5$ & $10.3 \pm 3.4$ \\
UV $(5 \mathrm{~s})$ & - & $5.1 \pm 3.1$ & $2.6 \pm 2.1$ \\
Vis $(60 \mathrm{~s})$ & - & $8.5 \pm 2.8$ & $5.8 \pm 2.4$ \\
\hline Before & $17.0 \pm 2.6$ & $12.7 \pm 3.9$ & $10.5 \pm 2.8$ \\
UV $(10 \mathrm{~s})$ & $9.6 \pm 3.3$ & $2.4 \pm 1.2$ & $2.3 \pm 1.1$ \\
Vis $(60 \mathrm{~s})$ & $14.5 \pm 2.3$ & $6.4 \pm 1.7$ & $4.9 \pm 1.8$ \\
\hline
\end{tabular}

Table S2. Average FRET efficiency and QD-DAE distance upon different UV irradiation time.

\begin{tabular}{ccccc}
\hline & \multicolumn{2}{c}{ FRET efficiency (\%) } & \multicolumn{2}{c}{ QD-DAE distance (nm) } \\
& UV 5 s & UV 10 s & UV 5 s & UV 10 s \\
\hline Conc.1 & - & $44.1 \pm 17.3$ & - & $2.32 \pm 0.28$ \\
Conc.2 & $61.7 \pm 21.6$ & $79.2 \pm 14.0$ & $2.07 \pm 0.37$ & $1.75 \pm 0.25$ \\
Conc.3 & $75.4 \pm 14.0$ & $75.0 \pm 16.4$ & $1.82 \pm 0.24$ & $1.82 \pm 0.28$ \\
\hline
\end{tabular}

\section{References}

1. Talapin, D. V.; Rogach, A. L.; Kornowski, A.; Haase, M.; Weller, H., Highly Luminescent Monodisperse CdSe and CdSe/ZnS Nanocrystals Synthesized in a Hexadecylamine-Trioctylphosphine Oxide-Trioctylphosphine Mixture. Nano Lett. 2001, 1, 207-211.

2. Talapin, D. V.; Nelson, J. H.; Shevchenko, E. V.; Aloni, S.; Sadtler, B.; Alivisatos, A. P., Seeded Growth of Highly Luminescent CdSe/CdS Nanoheterostructures with Rod and Tetrapod Morphologies. Nano Lett. 2007, 7, 2951-2959.

3. Xie, R. G.; Kolb, U.; Li, J. X.; Basche, T.; Mews, A., Synthesis and Characterization of Highly Luminescent CdSe-Core $\mathrm{Cds} / \mathrm{Zn}_{0.5} \mathrm{Cd}_{0.5} \mathrm{~S} / \mathrm{ZnS}$ Multishell 
Nanocrystals. J. Am. Chem. Soc. 2005, 127, 7480-7488.

4. Maus, M.; Cotlet, M.; Hofkens, J.; Gensch, T.; De Schryver, F. C.; Schaffer, J.; Seidel, C. A. M., An Experimental Comparison of the Maximum Likelihood Estimation and Nonlinear Least Squares Fluorescence Lifetime Analysis of Single Molecules. Anal. Chem. 2001, 73, 2078-2086.

5. Irie, M.; Lifka, T.; Uchida, K.; Kobatake, S.; Shindo, Y., Fatigue Resistant Properties of Photochromic Dithienylethenes: By-Product Formation. Chem. Commun. 1999, 747-748. 